\title{
Estudio de factibilidad de un diseño web para el registro, control y seguimiento de los sílabos académicos en las Universidades.
}

Feasibility study of a web design for the registration, control and monitoring of the academic syllables in the Universities.

Luis Alfonso Núñez Freire. ${ }^{1}$, Miguel Mesias Moreta Chávez. ${ }^{2}$, Nexar Ignacio Baque Yoza. ${ }^{3}$, Gerardo Alfredo Solano Gutiérrez. ${ }^{4}$, Juan Carlos Sarmiento Saavedra. ${ }^{5}$ \& Ramiro Enrique Guaman Chávez. ${ }^{6}$

Recibido: 10-10-2018 / Revisado: 17-11-2018 /Aceptado: 04-12-2018/ Publicado: 05-01-2019

\begin{abstract}
.
DOI: https://doi.org/10.33262/cienciadigital.v3i1.280

This research is carried out in order to carry out a feasibility study on a web system implementation to record, control and follow up the syllabi for an adequate control that can be made on the academic progress and, on the planning of the teaching units, it depends to a great extent the success or achievement of the proposed objectives both university headquarters located in the province of Esmeraldas and the director of the university headquarters in La Concordia. The analysis made to the control process that takes place in the educational community, made it possible to show that information systems are managed using manual processes, spreadsheets and even non-standardized procedures that make the control that can be carried out over academic and Class plans can not be made, using free software tools and applying the design standards proposed by Software Engineering, by implementing the system in the institution's computer network, within a Client-Server architecture. it has managed to improve the control of the compliance of the Syllables and the curriculum planning of the classroom, improving the processes and automating the procedures in a new digital information system.
\end{abstract}

1 Universidad Técnica Luis Vargas Torres de Esmeraldas, Ecuador alfnunez@gmail.com

2 Universidad Técnica Luis Vargas Torres de Esmeraldas, Ecuador, saisem_m@hotmail.com

3 Universidad Técnica Luis Vargas Torres de Esmeraldas, Ecuador, ney_baque01@ @otmail.com

4 Universidad Técnica Luis Vargas Torres de Esmeraldas, Ecuador, a_solano_g@ hotmail.com

5 Universidad Técnica Luis Vargas Torres de Esmeraldas, Ecuador, samientoj@gmail.com

6 Universidad Técnica Luis Vargas Torres de Esmeraldas, kenry.eg@ hotmail.com 
Keywords: Software, academic planning, Documentary information systems, Academy

\section{Resumen.}

Esta investigación se realiza con el fin hacer un estudio de factibilidad sobre una implementación de sistema web para registrar, controlar y dar seguimiento al sílabo para un adecuado control que se pueda realizar sobre el avance académico y, sobre la planificación de las unidades didácticas, depende en gran medida el éxito o la consecución de los objetivos propuestos tanta universidad matriz ubicado en la provincia de Esmeraldas y el director de la sede universitaria en La Concordia. El análisis realizado al proceso de control que se efectúa en la comunidad educativa, permitió evidenciar que se manejan sistemas de información que utilizan procesos manuales, hojas de cálculo e incluso procedimientos no estandarizados que hacen que el control que se pueda llevar sobre el avance académico y los planes de clase, no se pueden realizar, usando herramientas de software libre y aplicando los estándares de diseño que propone la Ingeniería de Software, mediante la implementación del sistema en la red informática de la institución, dentro de una arquitectura ClienteServidor, se ha lograra mejorar el control del cumplimiento del Sílabos y la planificación del currículo del aula, mejorando los procesos y automatizando los procedimientos en un nuevo sistema de información digital.

Palabras claves: Software, planificación académica, Sistemas de información documental, Academia.

\section{Introducción.}

Las instituciones de educación superior, para cumplir y ejecutar las cátedras universitarias y desempeñar su rol de formadores en educación deben desarrollar planificaciones curriculares como, sílabos, planes de clase y seguimiento al cumplimiento del sílabo, por lo tanto, están obligados y encargados de seguir la programación, preparar la evaluación y contribuir al inter- aprendizaje apoyados en las didácticas (Drago , 2017).

La Universidad Luis Vargas Torres sede La Concordia al ser una de las instituciones del estado y más importante de la región, contribuye al desarrollo y formación de miles de jóvenes de diferentes provincias. Los syllabus, es uno de los instrumentos fundamentales para realizar de una manera sistemática el trabajo del profesor en todo el ciclo académico por cada una de las materias que imparte, con el fin de realizar con más eficacia cada uno de los procesos, metodología, técnicas, instrumentos y evaluación que tiene el estudiante dentro de su área o entorno del aula (De la Torre, Oliver , \& Sevillano, 2010).

Con este propósito y conociendo que los docentes contribuyen en forma positiva al aprendizaje en las instituciones superiores, se aspira coadyuvar a la gestión que desarrollan 
las instituciones al ejercer un control sobre el trabajo que realizan los docentes en relación al desarrollo del contenido académico que se imparte en clases (Sílabos), (Macedo, 2012).

En la actualidad, la institución cuenta con un sistema de control de Sílabos en base a procesos dispersos, entregas en digital, hojas de Excel, Word, hojas de registro (leccionarios), que son llenados por los docentes con datos confusos que no coinciden con el programa académico debido a que el leccionario no siempre se encuentra en el aula por la falta de organización de los responsables académicos (Puebla , 2014). Por esta falta de organización y registros inadecuados en la sede universitaria requiere la automatización para el registro y seguimiento de los contenidos académicos que los docentes imparten en cada asignatura, para que los seguimientos diarios se ingresen de manera correcta y con datos reales, cosa que no sucede en la actualidad con el uso de los leccionarios (Jere, Hasbún, \& Rittershaussen, 2015).

Mediante el uso de una herramienta web desarrollada para llevar el seguimiento y cumplimiento de los sílabos de los docentes, el sistema llamado Lightswitch HTML, que sería usado de aplicación en la educación (Naranjo \& Urrutia, 2016). Esta aplicación web servirá para el control y registro de los sílabos, de esta manera eliminar la documentación que conlleva que cada docente que entrega impresa al departamento académico de la institución, optimizando tiempo y recursos, para tener así información eficiente y veraz, la aplicación web será desarrollada bajo software libre, que utilizará para su diseño la metodología de Proceso racional unificado también conocido por sus siglas (RUP), compuesto de cuatro fases denominadas (Rocafuerte \& Figeroa , 2016):

- Incepción o inicio

- Elaboración

- Desarrollo o Construcción

- Transición o transición.

Lo que permite un desarrollo confiable en lo que ha modelado de sistemas se refiere. Se utilizará además las herramientas UML (lenguaje unificado de modelado) para realizar los diagramas que servirán de apoyo en el desarrollo del proyecto. Se utilizará la plataforma de desarrollo WAMP Para el desarrollo y construcción del sistema (Naranjo \& Urrutia, 2016).

- Windows, como sistema operativo;

- Apache, como servidor web;

- MySQL, como gestor de bases de datos;

- PHP como lenguajes de programación.

El uso de este sistema de infraestructura denominado WAMP posibilita subir páginas HTML a internet y permite administrar en ellas. Además, un WAMP ofrece lenguajes de programación para desarrollar aplicaciones web. 
Mediante esta solución tecnológica la sede universitaria optimizará este proceso haciendo uso de la tecnología que en la actualidad exige el mundo moderno. Tiene una relevancia social con los beneficiados, que serán el departamento académico y los docentes de esta institución, porque contarán con una herramienta donde podrán realizar seguimiento de sus avances planificados (Villalobos, 2003). El desarrollo de esta investigación asiste a uno de los procesos fundamentales que tiene la sede universitaria y que con esta aplicación se podrá obtener información más fiable, lo que permitirá emitir reportes después de que los avances sean grabados, permitiendo así disminuir las 10 horas que es el tiempo que emplean entre el control, registro y seguimiento del avance académico, en comparación de un aproximado de 3 horas (en todos los procesos) con el uso del sistema y que a su vez permitirá reducir gastos.

\section{Método y materiales}

Planificar una estrategia para un trabajo investigativo que involucre el desarrollo de sistemas informáticos, y la implementación de los mismos en situaciones reales, debe ser un aspecto fundamental en el proceso de consolidación de los grupos y líneas de investigación, por tal motivo, se obtienen en primera instancia la información obtenida por las observaciones realizadas y las entrevistas informales, las cuales dieron las pautas necesarias para la elaboración de la encuesta. Cabe destacar que el desarrollo de la investigación está enmarcado como una investigación mixta, es decir, de campo y documental. Para acercarse a las características del problema en estudio y extraer de ellas información, se vale de cualquier recurso que le permita alcanzar sus objetivos.

En cuanto a la técnica utilizada para la obtención de la muestra representativa, se utilizó el muestreo intencional donde se eligen los individuos representativos de la población que interviene en el diseño y control del sílabo. se sigue el criterio del investigador para seleccionar a los sujetos que puedan facilitar la información necesaria, de tal manera que se considera todo el conjunto universo objeto del presente trabajo de investigación, conformado por 41personas entre ellos 1 Directivos General, un Director Académico, un Administrativo 48 docentes de la sede universitaria La Concordia.

\section{Análisis de resultados}

Determinar la factibilidad y aceptación del sistema propuesto por parte de los docentes y personal administrativo de la Sede universitaria a continuación detallaremos los resultados.

Tabla 1. ¿Participa Ud. de manera directa en la elaboración de los sílabos correspondientes a una determinada materia? 


\section{FRECUENCIA CANTIDAD PORCENTAJE}

\begin{tabular}{lcc}
\hline Siempre & 39 & $95 \%$ \\
Algunas Veces & 2 & $5 \%$ \\
Nunca & 0 & \\
$\quad$ TOTAL & 41 & $100 \%$ \\
\hline
\end{tabular}

Elaborado por: Grupo Investigador

La participación del personal encuestado sobre el proceso de elaboración del sílabo, se puede observar en un alto porcentaje. Esto es un indicador importante de que los principales actores del presente trabajo de investigación son los profesores con un 95\% de participación. Con respecto al 5\%, se pudo constatar que estos corresponden a personal que no está totalmente involucrado en el tema.

Tabla 2. ¿Cómo estima la carga de trabajo en la elaboración de los syllabus en la actualidad?

\begin{tabular}{lcc}
\hline FRECUENCIA & CANTIDAD & PORCENTAJE \\
\hline Alta & 35 & $85 \%$ \\
Media alta & 2 & $5 \%$ \\
Media & 2 & $5 \%$ \\
Baja & 2 & $5 \%$ \\
\multicolumn{1}{r}{ TOTAL } & 41 & $100 \%$ \\
\hline
\end{tabular}

Elaborado por: Grupo Investigador

La mayoría de los encuestados manifiestan el $85 \%$ afirma que la carga de trabajo para la elaboración del sílabo consume muchos recursos materiales y tiempo, debido a procesos manuales que deben ser desarrollados en el sistema de información actual, esto representa una carga alta de trabajo, nuevamente el porcentaje minoritario lo conforma personal que no participan en la elaboración del syllabus.

Tabla 3. Considera que el control que se lleva con respecto al cumplimiento de la planificación del syllabus es:

\begin{tabular}{lccc}
\hline FRECUENCIA & CANTIDAD & PORCENTAJE \\
\hline Muy adecuada & 0 & & - \\
Adecuada & 0 & & - \\
Poco adecuada & 2 & $5 \%$ & \\
No es adecuada & 39 & $95 \%$ & \\
\multicolumn{1}{r}{ TOTAL } & 41 & $100 \%$ & \\
\hline
\end{tabular}


Elaborado por: Grupo Investigador

Se ha evidenciado una inconformidad por parte de los encuestados, al momento de ser consultados en el diseño y evaluación del cumplimiento del sílabo, consideran que el control actual no se encuentra debidamente implementado, ya que no se considera ciertos factores que merman el trabajo de los docentes, sobre todo por la amplitud del currículo que deben cumplir, de los docentes encuestados el 95\% de ellos no están de acuerdo con el proceso de control actual.

Tabla 4. la generación de informes respecto al cumplimiento de la planificación del syllabus le representa una carga de trabajo:

\begin{tabular}{|c|c|c|}
\hline FRECUENCIA & CANTIDAI & PORCENTAJE \\
\hline Alta & 35 & $85 \%$ \\
\hline Media alta & 4 & $10 \%$ \\
\hline Media alta & 1 & $2 \%$ \\
\hline Baja & 1 & $2 \%$ \\
\hline TOTAL & 41 & $100 \%$ \\
\hline
\end{tabular}

Elaborado por: Grupo Investigador

Referente a la carga de trabajo que representa la elaboración de informes sobre el cumplimiento de la planificación de los syllabus, el $86 \%$ de los encuestados nos indicaron que la carga de trabajo es alta, los resultados indican que la elaboración de informes le agrega una carga de trabajo adicional a las actividades inherentes al cumplimiento del currículo, sobre todo para aquellos docentes que no dominan las TICS, y deben utilizar procesos manuales.

Tabla 5. Considera que deberían existir otros mecanismos para el control del cumplimiento académico por parte de los docentes, cuáles estimas convenientes.

\begin{tabular}{lcc}
\hline \multicolumn{1}{c}{ FRECUENCIA } & CANTIDAD & PORCENTAJE \\
\hline sistemas informáticos & 20 & $49 \%$ \\
Libro Excel & 5 & $12 \%$ \\
Bases de datos & 15 & $37 \%$ \\
Me es indiferentes & 1 & $2 \%$ \\
$\quad$ TOTAL & 41 & $100 \%$ \\
\hline
\end{tabular}

Elaborado por: Grupo Investigador 
Estos resultados nos servirán para determinar las preferencias del personal docente y administrativo hacia la utilización de mecanismos de control con el empleo de las Tecnologías de la Información, del análisis obtenido, se observa que el $49 \%$ de los encuestados, consideran que la intervención de la implementación de una solución tecnológica en el proceso de control del syllabus es necesaria el porcentaje minorista corresponde a encuestados que aún mantienen brechas tecnológicas.

Tabla 6. Participaría de manera activa en la propuesta de implementación de una solución informática para mejorar el control del syllabus:

\begin{tabular}{|c|c|c|}
\hline FRECUENCIA & CANTIDAD & PORCENTAJE \\
\hline $\mathrm{Si}$ & 38 & $93 \%$ \\
\hline No & 0 & - \\
\hline No dominio de las TIC & 3 & $7 \%$ \\
\hline Me es indiferentes & 0 & - \\
\hline TOTAL & 41 & $100 \%$ \\
\hline
\end{tabular}

Elaborado por: Grupo Investigador

El 93\%, de los encuestados afirmaron que participarían activamente para la consecución satisfactoria del proyecto, estos resultados nos demuestran que la implementación de un sistema para el control y seguimiento de los sílabos de docentes Universidad Técnica Luis Vargas Torres de Esmeraldas Sede La Concordia " gozaría de gran aceptación tanto de personal docente como administrativo de la Institución, y se convierte en una oportunidad para determinar la factibilidad de implementación del sistema propuesto.

Tabla 7. Considera que debería existir una herramienta tecnológica que facilite el control del contenido académico y la generación de informes de manera automática:

\begin{tabular}{lcc}
\hline \multicolumn{1}{c}{ FRECUENCIA } & CANTIDAD & PORCENTAJE \\
\hline Muy de acuerdo & 39 & $95 \%$ \\
No es necesario & 0 & - \\
Me es indiferentes & 2 & $5 \%$ \\
$\quad$ TOTAL & 41 & $100 \%$ \\
\hline
\end{tabular}

Elaborado por: Grupo Investigador

El 95\% de los docentes opinan que es necesaria la existencia de un sistema informático que permita facilitar el control del cumplimiento y la generación automática de informes y de esta manera se puedan minimizar el uso excesivo de recursos humanos, materiales y tiempo, implementados en el diseño, evaluación y control del syllabus, incluso, los docentes que 
mantienen brechas tecnológicas también consideran ser parte de este proceso, mientras que el $5 \%$ le es indiferentes que exista un sistema.

Tabla 8. Estaría dispuesto a cambiar la forma tradicional de gestión de los planes de clases mediante el uso de un sistema informático y base de datos:

\begin{tabular}{lcc}
\hline FRECUENCIA & CANTIDAD & PORCENTAJE \\
\hline Muy de acuerdo & 35 & $85 \%$ \\
No es necesario & 4 & $10 \%$ \\
Me es & & $5 \%$ \\
indiferentes & 2 & $100 \%$ \\
\multicolumn{1}{r}{ TOTAL } & 41 & \\
\hline
\end{tabular}

Elaborado por: Grupo Investigador

En cuanto a la resistencia al cambio en la manera tradicional de llevar la información, el 85\% de los encuestados, está de acuerdo en la utilización de un sistema informático con base de datos que permita mejorar procesos y evitar errores que se comenten en la actualidad; ya que la mayoría de procesos son llevados de forma manual lo que representa un esfuerzo adicional dentro del proceso de cumplimiento en sílabo, la injerencia que tiene la sociedad de la información, deben ser aprovechadas en la sede universitaria y un $10 \%$ dice que no es necesario cambiar la estructura existente mientras que 5\% no le afectaría en nada la utilización tanto del sistema o hacerlo manualmente.

Tabla 9. Para el caso de los sistemas informáticos que ayudan en la gestión del sillabos, cuáles consideras que debería ser los lugares de acceso al mismo:

\begin{tabular}{lcc}
\hline FRECUENCIA & CANTIDAD & PORCENTAJE \\
\hline Aula & 20 & $49 \%$ \\
Sala docente & 15 & $37 \%$ \\
Casa & 5 & $12 \%$ \\
Me es indiferente & 1 & $2 \%$ \\
\multicolumn{1}{r}{ TOTAL } & 41 & $100 \%$ \\
\hline
\end{tabular}

Elaborado por: Grupo Investigador

Al consultarle al personal participante de la encuesta a la Sede universitaria acerca de sus preferencias, del lugar de acceso al sistema informático para el control del sílabos, nos respondieron de la siguiente manera: $49 \%$ optan por ingresar a la aplicación en el aula de clase de la institución, 37\% eligieron el acceso desde la sala docente, mientras que $13 \%$ prefieren acceder desde la casa y el $2 \%$ le es indiferente la manera de ingresar al sistema, lo que indica que no mantienen preferencia específica por ninguno de los lugares mencionados anteriormente. En base a las respuestas obtenidas podemos notar que los usuarios finales 
necesitarán diferentes alternativas de tal manera que ellos elijan la que mejor se adapte a sus necesidades.

\section{Discusión}

De los resultados obtenidos se identificó que principales actores en el diseño y elaboración del sílabo son los profesores con un $95 \%$ de participación, de la misma manera al análisis de resultado nos muestra que hay un $85 \%$ de docentes que manifiestan que la carga de trabajo para la elaboración del syllabus es elevada debido a los procesos manuales que desarrollan.

Estas cargas de trabajo inciden en el cumplimento del currículo y de la planificación del aula, ya que existe un $95 \%$ de los docentes que manifiestan inconformidad con el proceso vigente. Esto también afecta la entrega de informes referente al seguimiento del cumplimiento de las unidades académicas, donde el $86 \%$ de los encuestados consumen mucho tiempo en la elaboración de los mismos. En cuanto a que medios les gustarían a los actores del proceso de elaboración y control de sílabos, la mayoría de los encuestados coinciden en que se deberían implementar soluciones tecnológicas como sistemas informáticos y bases de datos, solo el $12 \%$ prefiere mantenerse con libros de Excel.

Además, se afirman que participarían en la implementación de un sistema para el control y seguimiento de los sílabos de docentes en la Universidad Técnica Luis Vargas Torres de Esmeraldas Sede La Concordia el cual permitiría la gestión eficiente de estos procesos.

\section{Conclusiones}

- En el análisis preliminar del control del sílabo, se logró constatar un promedio de tiempo de procesos de 10 horas en la revisión y control del syllabus de las asignaturas, al implementar la solución tecnológica en ambiente web, se pudo reducir estos tiempos en un $70 \%$ aproximadamente, permitiendo una gestión más eficiente.

- Mediante la implementación del paquete de instalación wampp con herramientas open source, se diseñará la aplicación web su inversión es rentable y accesible que no requiere de un alto costo de inversión.

- El estudio de factibilidad del diseñó un modelo de datos relacional, el cual va almacenar la información pertinente al sistema de control de sílabos, aplicando políticas de seguridad en el acceso al software y hardware que contienen los datos de la aplicación, mejorando en un $80 \%$ las vulnerabilidades que mantenía el anterior sistema de información. 


\section{Referencias Bibliográficas.}

De la Torre, S., Oliver , C., \& Sevillano, L. (2010). Estrategia didáctica en el aula:Buscando la calidad y la Innovación. Madrid: GIAD-EDIFI. Obtenido de R. A. MARINO, Conectados en el ciberespacio, Madrid: UNED Editorial, 2010.

Drago , C. (2017). Manual de Apoyo Docente. Santiago - Chile: Universidad Central. Obtenido de http://www.ucentral.cl/prontus_ucentral2012/site/artic/20170830/asocfile/20170830 100642/manual_evaluacion.pdf

Jere, O., Hasbún, B., \& Rittershaussen, S. (2015). El diseño de un Syllabus en la eudcacion supeiror una propuesta metodológica (Primera ed.). Santiago - Chile: Universidad de Chile. Obtenido de https://www.plataforma.uchile.cl/libros/SYLLABUS_01_dic.pdf

Macedo, V. (2012). Diagnostico de clima organizacional en el Centro Universitario de los Valles. revista Iberoamericana para la Investigación y el Desarrollo Educativo, 1(8), 10. Obtenido de http://ride.org.mx/111/index.php/RIDESECUNDARIO/article/viewFile/8/8

Naranjo , T., \& Urrutia, E. (16 de agosto de 2016). Sistema Web para el seguimiento del cumplimiento de los contenidos de sílabos en la Facultad de Ingeniería en Sistemas, Electrónica e Industrial. Tesis de pregrado Univerisdad de Tècnica de Ambato. Ambato. Obtenido de http://repositorio.uta.edu.ec/handle/123456789/23657

Puebla , A. (03 de mayo de 2014). La Investigacion Edicativa y su importancia en la practica docente que se desarrolla en la Escuela Nacional de Trabjo Social. Recuperado el 20 de septiembre de 2018, de Academia: https:/www.transformacioneducativa.com/index.php/articulos-sobre-educacion/54-importancia-de-lainvestigacion-educativa

Rocafuerte , J., \& Figeroa , E. (2016). Análisis Del Proceso y Sistematización Del Registro De Syllabus De Las Carreras de la Universidad De Guayaquil. Tesis de Pregrado Universidad de Guayaquil - Ecuador. Guayaquil. Obtenido de http://repositorio.ug.edu.ec/bitstream/redug/17587/1/UG-FCMF-B-CISCPTG.1064.pdf

Villalobos, J. (2003). El docente y actividades de enseñanza / aprendizaje: algunas consideraciones teóricas y sugerencias prácticas. Revista Educere, 7(22), 170-176. Obtenido de http://www.redalyc.org/pdf/356/35602206.pdf

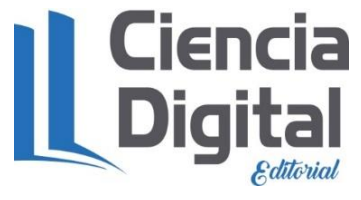


Para citar el artículo indexado.

Núñez L., Moreta M., Baque N., Solano G., Sarmiento J. \& Guaman R. (2019). Estudio de factibilidad de un diseño web para el registro, control y seguimiento de los sílabos académicos en las Universidades. Revista electrónica Ciencia Digital 3(1), 190-200. Recuperado desde: http://cienciadigital.org/revistacienciadigital2/index.php/CienciaDigital/article/view/280/67 $\underline{6}$

\section{¿Ciencia}

El artículo que se publica es de exclusiva responsabilidad de los autores y no necesariamente reflejan el pensamiento de la Revista Ciencia Digital.

El artículo queda en propiedad de la revista y, por tanto, su publicación parcial y/o total en otro medio tiene que ser autorizado por el director de la Revista Ciencia Digital.
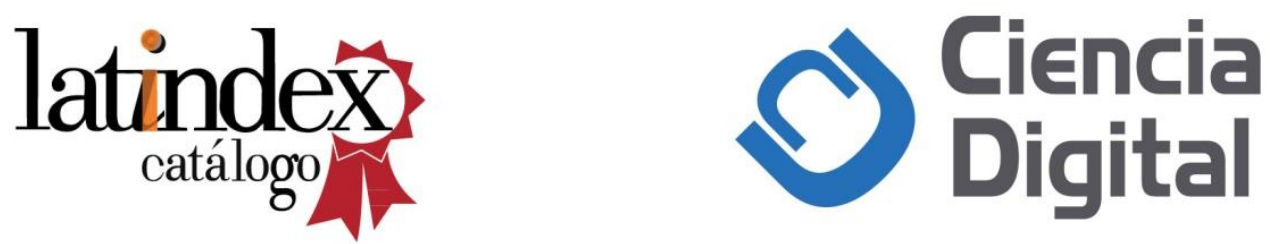